\title{
IncRNA NEAT1 regulates the proliferation and migration of hepatocellular carcinoma cells by acting as a miR-320a molecular sponge and targeting $L$ antigen family member 3
}

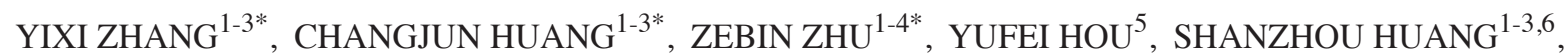 \\ CHENGJUN SUN ${ }^{1-3}$, YUNHUA TANG ${ }^{1-3}$, ZHIHENG ZHANG ${ }^{1-3}$, LINHE WANG $^{1-3}$, \\ HUADI CHEN $^{1-3}$, WEIQIANG JU ${ }^{1-3}, \mathrm{XIN}$ QIAO $^{7}$ and MAOGEN CHEN ${ }^{1-3}$ \\ ${ }^{1}$ Organ Transplant Center, The First Affiliated Hospital, Sun Yat-sen University; \\ ${ }^{2}$ Guangdong Provincial Key Laboratory of Organ Donation and Transplant Immunology,
}

Department of Science and Technology of Guangdong Provincial, Ministry of Science and Technology of China; ${ }^{3}$ Guangdong Provincial International Cooperation Base of Science and Technology, Guangzhou, Guangdong 510080;

${ }^{4}$ Organ Transplant Center, The First Affiliated Hospital, Division of Life Sciences and Medicine, University of Science and Technology of China, Hefei, Anhui 230001; ${ }^{5}$ Department of Ultrasound Medicine, The First Affiliated Hospital of

Guangdong Pharmaceutical University, Guangzhou, Guangdong 510600; ${ }^{6}$ Department of General Surgery,

Guangdong General Hospital, Guangdong Academy of Medical Sciences, Guangzhou, Guangdong 510000;

${ }^{7}$ Department of Anesthesiology, Beijing Chaoyang Hospital, Capital Medical University, Beijing 100020, P.R. China

Received July 18, 2019; Accepted April 2, 2020

DOI: 10.3892/ijo.2020.5108

\begin{abstract}
Long non-coding RNAs (lncRNAs) serve a pivotal role in hepatocellular carcinoma (HCC) progression and have been confirmed to participate in the carcinogenesis and development of HCC. Certain studies have focused on lncRNA nuclear enriched abundant transcript 1 (NEAT1) in HCC. However, the relationship between IncRNA NEAT1 and HCC remains unclear. The present study found that NEAT1 was significantly overexpressed in HCC cell lines compared with LX-2 hepatic stellate cells. NEAT1 expression in Huh7 and MHCC-97H cells was increased following transfection
\end{abstract}

Correspondence to: Dr Xin Qiao, Department of Anesthesiology, Beijing Chaoyang Hospital, Capital Medical University, 8 Gongren Tiyuchang South Road, Beijing 100020, P.R. China

E-mail: 1365356493@qq.com

Dr Maogen Chen, Organ Transplant Center, The First Affiliated Hospital, Sun Yat-sen University, 58 Zhongshan Er Road, Guangzhou, Guangdong 510080, P.R. China

E-mail: maogen2000@163.com

*Contributed equally

Abbreviations: lncRNA, long noncoding RNA; HCC, hepatocellular carcinoma; RT-qPCR, reverse transcription-quantitative PCR

Key words: long non-coding RNA nuclear enriched abundant transcript 1, L antigen family member 3, micorRNA-320a, proliferation, migration, hepatocellular carcinoma with lentivirus (LV)-NEAT1 but inhibited by LV-short hairpin NEAT1. Knockdown of NEAT1 significantly repressed HCC cell viability, increased cell apoptosis, and inhibited cell migration and invasion capacity. By contrast, upregulation of NEAT1 demonstrated the reverse effects. Furthermore, microRNA-320a (miR-230a) was predicted to be a direct target of NEAT1 and was significantly reduced in HCC cells. Additionally, a luciferase activity reporter assay and RNA immunoprecipitation assay were performed to confirm the interaction between miR-320a and NEAT1. Using a dual-luciferase activity assay, L antigen family member 3 (LAGE3) was found to be a target of miR-320a. Finally, in vivo nude mouse models were established, and the results indicated that NEAT1 suppressed HCC progression by targeting miR-320a. In conclusion, the present findings revealed that the NEAT1/miR-320a/LAGE3 axis participates in HCC development and that NEAT1 could be used as a biomarker for HCC.

\section{Introduction}

Hepatocellular carcinoma (HCC) is the most common malignant tumor type of the liver and originates from the malignant transformation of hepatocytes; it accounted for $75-85 \%$ of primary liver cancers in 2018 worldwide $(1,2)$. Resection, transarterial chemoembolization, radiofrequency ablation, liver transplantation, selective internal radiation therapy and systemic therapies are considered effective methods for treating patients with different stages of HCC (3). However, the trend of increasing HCC-associated mortality remains a growing concern (4). Therefore, a better understanding of the molecular mechanisms related to HCC progression would be favorable for early diagnosis and effective management. 
Long non-coding RNAs (lncRNAs) are molecules containing $>200$ nucleotides that do not code for proteins (5). The molecular functions of lncRNAs include transcriptional splicing, chromosome structure regulation, mRNA stability, mRNA availability and posttranslational modification (6). MicroRNAs (miRNAs) are a class of evolutionarily conservative non-coding RNAs comprised of $\sim 22$ nucleotides that have an essential role in posttranscriptional gene regulation (7). They can bind to numerous sites (nucleotides 2-7), particularly those in the 3'-untranslated region of target gene mRNAs, and modulate target gene expression by suppressing the translation of target mRNAs (8). Both lncRNAs and miRNAs serve key roles in numerous biological processes, including the immune response, cell growth, epigenetic regulation, tumorigenesis and cell differentiation $(9,10)$. Recently, increasing evidence has suggested that the crosstalk between lncRNAs and miRNAs plays essential roles in the development and progression of numerous cancers, including HCC $(11,12)$. For instance, the lncRNA FTX contributes to colorectal cancer tumorigenesis and progression via an interaction with miR-215 (13). The IncRNA TUG1 modulates the progression of oral squamous cell carcinoma by sponging miR-524-5p and mediates the expression of distal-less homeobox 1 (DLX1) (14). In addition, the IncRNA CASC2 regulates the development of HCC cells via the miR-362-5p/NF- $\kappa$ B axis (15).

NEAT1 is a type of IncRNA that is abnormally expressed in HCC and has various oncogenic roles (16-18). Furthermore, it is generally correlated with the progression of several types of neoplasms, such as colorectal cancer, breast cancer and non-small cell lung cancer (19-21). Yan et al (22) reported that NEAT1 enhances the resistance of anaplastic thyroid carcinoma cells to cisplatin by sponging miR-9-5p and regulating sperm associated antigen 9 expression. Xia et al (23) reported that NEAT1 promotes the growth of gastric cancer cells by regulating the miR-497-5p/phosphoinositide-3-kinase regulatory subunit 1 axis. Furthermore, NEAT1 can promote malignant melanoma development and metastasis by targeting ras-releated protein Rab-9 (24). The oncogenic function of NEAT1 in HCC is gradually emerging; however, the detailed mechanism of HCC remains largely unknown.

The present study mainly focused on the biological function of NEAT1 in HCC. NEAT1 was upregulated and miR-320a was downregulated in HCC cells. HCC cell progression was significantly affected by NEAT1 silencing or overexpression. In addition, miR-320a was predicted as a target of NEAT1 and regulated the level of LAGE3. Furthermore, NEAT1 was upregulated in HCC tissues and positively correlated with LAGE3 expression. Therefore, it was hypothesized that NEAT1 contributes to the proliferation and migration of HCC by acting as a miR-320a molecular sponge and targeting LAGE3.

\section{Materials and methods}

Cell culture. Human hepatic carcinoma cell lines (Huh7, SNU-398, MHCC-97H, Hep3B and SNU-449), hepatoblastoma cells Huh-6, hepatic stellate cells LX-2 and 293T cells were used in the present study. The cells were all purchased from the Institute of Cell Biology, Chinese Academy of Sciences. RPMI-1640 (Sigma-Aldrich; Merck KGaA) or Dulbecco's modified Eagle's medium (DMEM; Sigma-Aldrich; Merck KGaA) supplemented with $10 \%$ fetal bovine serum (FBS; HyClone; GE Healthcare), $100 \mathrm{U} / \mathrm{ml}$ penicillin and $100 \mu \mathrm{g} / \mathrm{ml}$ streptomycin (Gibco; Thermo Fisher Scientific, Inc.) was used as the cell culture medium, and the cells were all cultured in a humidified chamber containing $5 \% \mathrm{CO}_{2}$ at $37^{\circ} \mathrm{C}$. Huh7 and MHCC-97H cell lines were cultured by RPMI-1640, while other cell lines were cultured by DMEM.

Lentiviral vector transfection. Lentiviral plasmids (lentivirus-shNEAT1) were constructed using short hairpin RNAs (shRNAs) of the NEAT1 sequence (Shanghai GenePharma Co., Ltd.), and were transfected into Huh7 and MHCC-97H cells using Lipofectamine ${ }^{\circledR} 2000$ reagent (Invitrogen; Thermo Fisher Scientific, Inc.). The concentration of lentiviral plasmid transfected was $2 \mu \mathrm{M}$. The lentiviral plasmid containing a scrambled sequence was constructed using the LV-NC (Shanghai GenePharma Co., Ltd.) as a negative control. The oligonucleotide sequences were: shNEAT1 sense, 5'-CACCGCATGGACCGTGGT TTCCGTTACTTTCAAGAGAAGTAACAAAACGGTCCA TGTTTTTTG-3' and antisense, 5'-GATCCAAAAAACATG GACCGTGGTTTGTTACTTCTCTTGAAAGTAACAAAC CACGGTCCATGC-3'; NC sense, 5'-CACCGTTCTCCG AACGTGTCACGTCAAGAGATTACGTGACACGTTCGG AGAATTTTTTG-3' and antisense, 5'-GATCCAAAAAAG TTCTCCGCGTGTCACGTAATCTCTTGACGTGACACG TTCGGAGAAC-3'. A synthetic and purified NEAT1 gene fragment (5'-CGGCUCGAGGGGCCAUCAGCUUU-3') was inserted into the lentiviral plasmids and designated LV-NEAT1 (Shanghai GenePharma Co., Ltd.).

The transfection of miR-320a mimic or negative control in Huh7 and MHCC-97H cell lines was performed using Lipofectamine $^{\circledR} 2000$ reagent (Invitrogen; Thermo Fisher Scientific, Inc.). The concentration of miR-320a mimic and negative control was $100 \mu \mathrm{M}$. The sequences of the miR-320a mimic and negative control were as follows: miR-320a mimic, 5'-AAA AGCUGGGUUGAGAGGGCGA-3' and negative control, 5'-UUCUCCGAACGUGUCACGUTT-3'. Further experiments were performed $48 \mathrm{~h}$ after transfection.

Transwell invasion assay. A 200- $\mu 1$ cell suspension of $1 \times 10^{5}$ Huh7 or MHCC-97H cells in RPMI-1640 medium was loaded into the upper chambers of a 24-well Transwell plate, which had an $8-\mu \mathrm{m}$ pore size and was coated with $1 \mathrm{mg} / \mathrm{ml}$ Matrigel (Corning Inc.) for $1 \mathrm{~h}$ at $4^{\circ} \mathrm{C}$. The lower chamber was loaded with $600 \mu 1$ RPMI-1640 containing 10\% FBS. Subsequently, the cells on the surface of the filter were fixed with $4 \%$ formaldehyde for $15 \mathrm{~min}$, stained with $0.5 \%$ crystal violet for $30 \mathrm{~min}$ at room temperature, and then counted using a light microscope (magnification, x200).

EdU proliferation assay. Cell proliferation was studied using the EdU proliferation assay (Nanjing Keygen Biotech Co., Ltd.) according to the manufacturer's instructions. A total of $48 \mathrm{~h}$ after transfection, $2 \times 10^{4}$ cells were treated with $10 \mu \mathrm{M}$ EdU solution for $4 \mathrm{~h}$. EdU-positive cells were observed using Apollo staining for $30 \mathrm{~min}$ at room temperature and 4',6-diamidino-2-phenylindole staining for $30 \mathrm{~min}$ at room temperature with an inverted fluorescence microscope (magnification, x200). Then, positive cells were identified 
Table I. Primer sequences used for reverse transcription-quantitative PCR.

\begin{tabular}{lll}
\hline Gene & \multicolumn{1}{c}{ Forward $\left(5^{\prime}-3^{\prime}\right)$} & \multicolumn{1}{c}{ Reverse $\left(5^{\prime}-3^{\prime}\right)$} \\
\hline NEAT1 & TGGCTAGCTCAGGGCTTCAG & TCTCCTTGCCAAGCTTCCTTC \\
miR-320a & GTTGGATCCGGCGTTTCCTTCCGACATG & GCTGAATTCGTCCACTGCGGCTGTTCC \\
LAGE3 & CGACTGTGGGTCAGTTTGCAC & GGTTGAGAGGCTGCGGTTT \\
MALAT1 & AAAGCAAGGTCTCCCCACAAG & GGTCTGTGCTAGATCAAAAGGCA \\
GAPDH & AAGGAAGGTGGTGAAGCAGGC & GTCAAAGGTGGAGGAGTGGG \\
U6 & ATTGGAACGGATACAGAGAAGATT & GGAACGCTTCACGAATTTG
\end{tabular}

NEAT1, nuclear enriched abundant transcript 1; miR-320a, microRNA-320a; LAGE3, L antigen family member 3.

by integral optical density (IOD) using Image-Pro Plus 6.0 (Media Cybernetics, Inc.).

Colony forming assay. LV-NEAT1-, LV-shNEAT1- and LV-NC-transfected Huh7 and MHCC-97H cells were seeded at a density of 100 cells/well in 6-well plates. After 2 weeks of culture, the colonies were fixed with methanol for $15 \mathrm{~min}$ at room temperature and stained with $0.5 \%$ crystal violet for $15 \mathrm{~min}$ at room temperature. Visible colonies were manually counted in randomly selected fields using light a microscope (magnification, x10). The clone formation rate (CFR) was calculated according to the following formula: $\mathrm{CFR}=$ clone counts/seeded cell counts $x 100 \%$. The experiment was repeated three times.

Flow cytometric analysis of cell apoptosis. Huh7 and MHCC-97H cell lines transfected with LV-NC, LV-shNEAT1 and LV-NEAT1 were stained using FITC-Annexin V and propidium iodide (PI; both Beyotime Institute of Biotechnology) in the dark for $15 \mathrm{~min}$ at room temperature. Apoptosis assays were performed using the CytoFLEX flow cytometer (Beckman Coulter, Inc.) and analyzed via Cytexpert 2.0 software (Beckman Coulter, Inc.). Annexin V-FITC-negative and PI-negative cells were defined as living cells; Annexin V-FITC-positive and PI-negative were defined as early apoptotic cells; Annexin V-FITC-positive and PI-positive were defined as late apoptotic cells and necrotic cells.

Reverse transcription-quantitative PCR (RT-qPCR). Total RNA form HCC tissues or cell lines was extracted using RNAiso Plus (Takara Bio, Inc.). To ensure purity of the total RNA, the $\mathrm{A}_{260} / \mathrm{A}_{280}$ of was controlled between 1.8 and 2.2, and the $A_{260} / A_{230}$ ratio was controlled $>1.7$. The total RNA concentration was between 500 and $1,000 \mathrm{ng} / \mu \mathrm{l}$. Prime Script $^{\mathrm{TM}}$ RT Master mix was used for RNA RT at $37^{\circ} \mathrm{C}$ for 15 min followed by $85^{\circ} \mathrm{C}$ for 5 sec. SYBR Premix Ex Taq II (Takara Bio, Inc.) was used for qPCR, which was performed on the Applied Biosystems 7500 Real-Time PCR system (Applied Biosystems; Thermo Fisher Scientific, Inc.). The following standard two-step PCR reaction program was used: i) one cycle of pre-denaturation at $95^{\circ} \mathrm{C}$ for $30 \mathrm{sec}$; ii) 40 cycles of $95^{\circ} \mathrm{C}$ for $5 \mathrm{sec}, 60^{\circ} \mathrm{C}$ for $20 \mathrm{sec}$; and iii) final extension at $72^{\circ} \mathrm{C}$ for $30 \mathrm{sec}$, followed by melting curve analysis and cool down. Relative gene expression levels were analyzed using the $2^{-\Delta \Delta \mathrm{Cq}}$ method (25). The primers of NEAT1, LAGE3, miR-320a and the controls are shown in Table I. GAPDH was used as a loading control to detect the NEAT1 and LAGE3 expression levels, and U6 was used as a loading control to detect the miR-320a expression level.

Bioinformatics analysis. ChipBase (http://rna.sysu.edu. cn/chipbase/index.php) and StarBase (http://starbase.sysu. edu.cn/) were used to identify specific miRNA targets of lncRNA NEAT1. The TargetScan Human 7.1 (http://www. targetscan.org/), Starbase and miRanda (http://www.microrna. org/microrna/home.do) databases were used to predict putative mRNA targets of miR-320a.

Luciferase reporter gene assay. For the luciferase reporter gene assay, $5 \times 10^{5} 293 \mathrm{~T}$ cells were inoculated in a 24 -well plate overnight. Subsequently, $150 \mathrm{ng}$ pmirGLO-LAGE3-WT or pmirGLO-lncRNA NEAT1-WT reporter plasmids (Promega Corporation) and their mutant vectors were co-transfected into cells with $50 \mathrm{nM}$ miRNA-320a mimic using Lipofectamine 2000 reagent. After $36 \mathrm{~h}$ of cell culture, the firefly and Renilla luciferase activity was determined by a double Luciferase Report Analysis system (Promega Corporation) based on the manufacturer's instructions. The relative luciferase activity was calculated based on the fluorescence of the firefly/Renilla luciferase.

RNA immunoprecipitation (RIP). The RIP assay was carried out utilizing a Magna RIP kit (EMD Millipore). Huh7 cells were lysed using RIP lysis buffer, and the cell lysates were incubated with magnetic beads conjugated to a human anti-Ago2 antibody (1:500; catalog no. MABE253; EMD Millipore). Huh7 cells were used to conduct the RIP assay without transfection prior to the assay. RNA was detected by RT-qPCR and IgG detected performed as a negative control. MALAT1 was selected as a positive control according to Sun et al (26), who revealed that MALAT1 acts as sponges of miR-320a. The primers for MALAT1 are presented in Table I.

RNA pull-down assay. RNA was purified and labeled with biotin using the Pierce RNA 3' End Desthiobiotinylation kit (Thermo Fisher Scientific, Inc.). Positive control (biotin-labeled wild-type miR-320a; miR-320a-Bio), negative control (mutant miR-320a, miR-320a-Bio-MUT), and biotinylated RNAs (NC-Bio) were incubated with the cell lysates. 

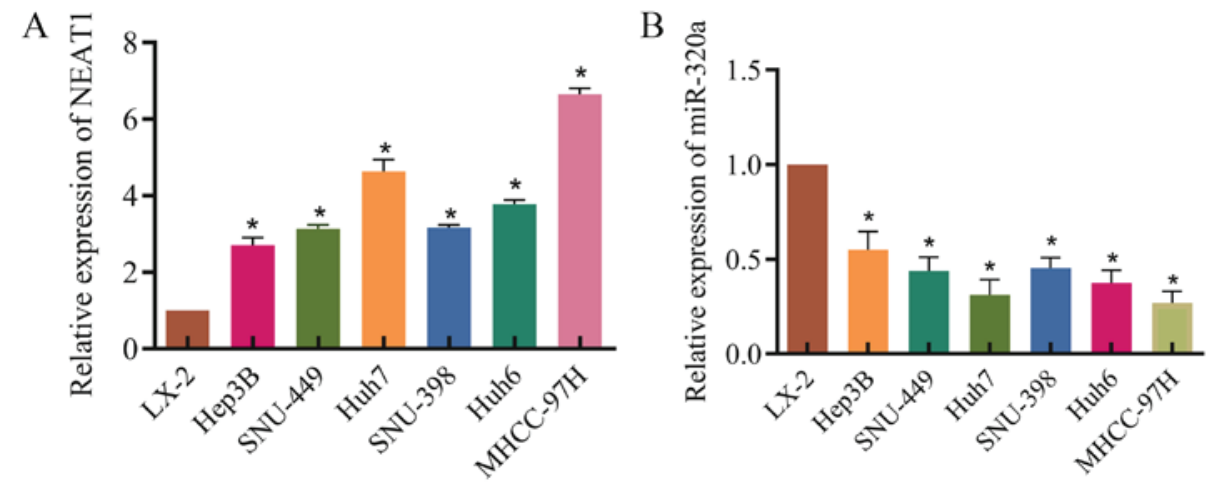

Figure 1. Expression of NEAT1 and miR-320a in hepatocellular carcinoma cells. (A) NEAT1 expression in LX-2, Hep3B, SNU-449, Huh6, SNU-398, Huh7 and MHCC-97H cells. RT-qPCR was used to detect NEAT1 expression, with GAPDH as a loading control. (B) miR-320a expression in LX-2, Hep3B, SNU-449, Huh6, SNU-398, Huh7 and MHCC-97H cells. RT-qPCR was used to test miR-320a expression, with U6 as a loading control. Three independent experiments were performed. "P<0.05 vs. LX-2. RT-qPCR, reverse transcription-quantitative PCR; miR-230a, microRNA-320a; NEAT1, nuclear enriched abundant transcript 1 .
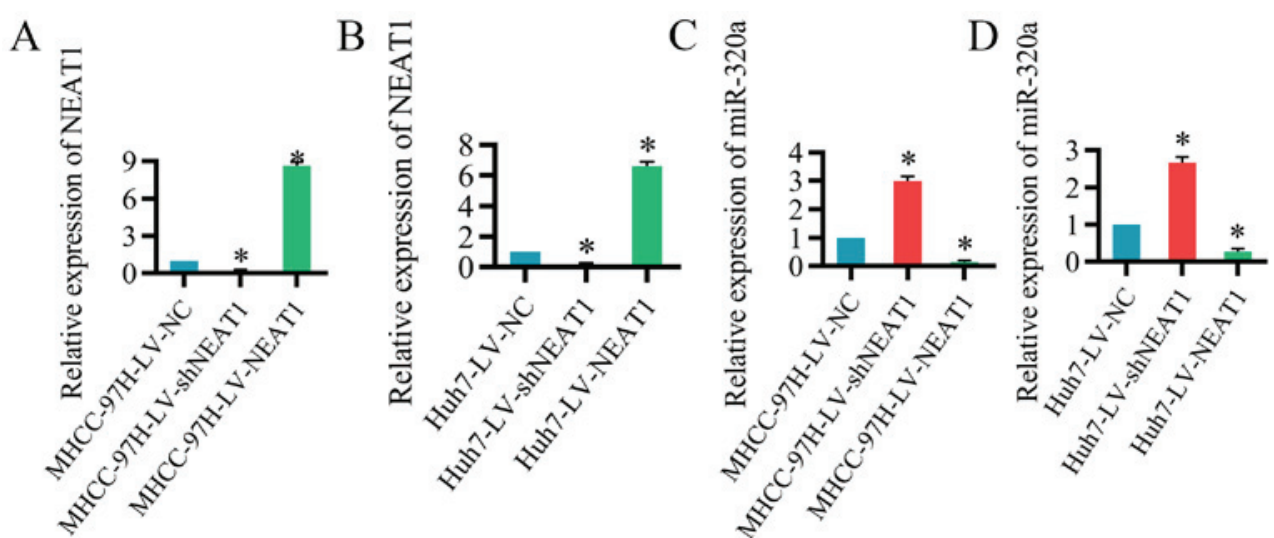

$\mathrm{E}$

DAPI

EdU

Merged
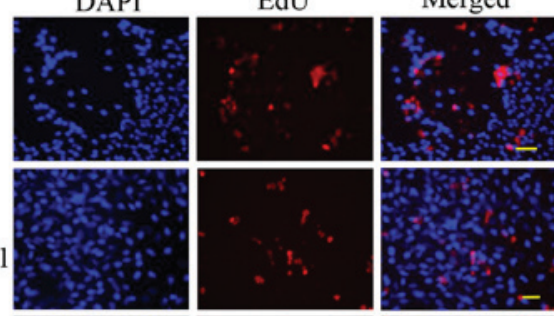

LV-shNEAT1
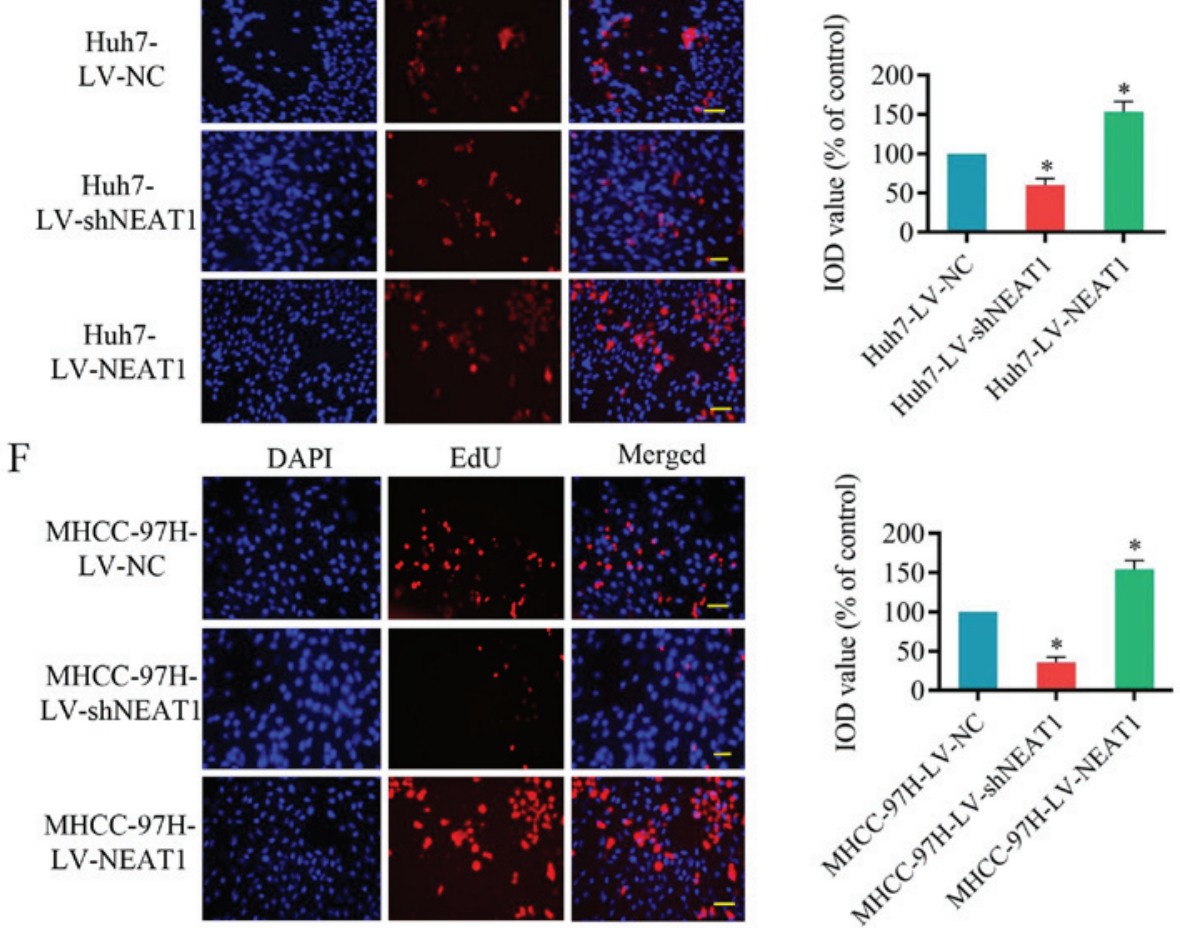

EdU
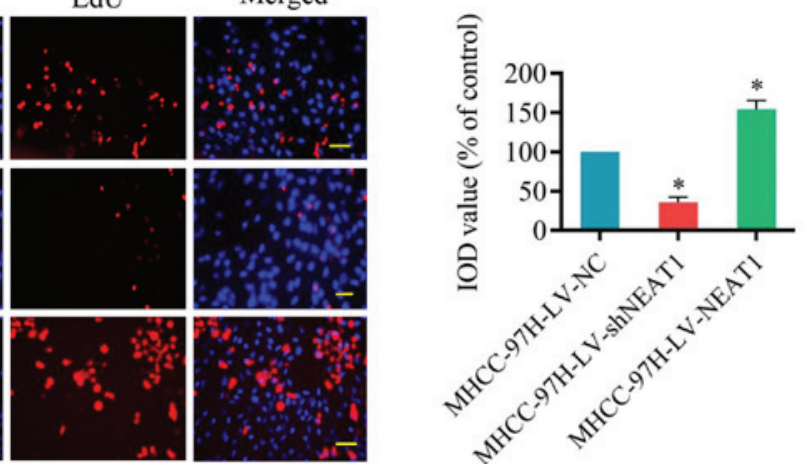

Figure 2. Effects of NEAT1 on hepatocellular carcinoma cell proliferation. Cells were infected with LV-shNEAT1 or LV-NEAT1 for $48 \mathrm{~h}$ prior to further analysis. NEAT1 expression in (A) MHCC-97H and (B) Huh7 cells. miR-320a expression in (C) MHCC-97H and (D) Huh7 cells. (E) Effects of NEAT1 on the proliferation of Huh7 cells. Scale bar, $50 \mu \mathrm{m}$. (F) Effects of NEAT1 on the proliferation of MHCC-97H cells. Scale bar, $50 \mu \mathrm{m}$. Three independent experiments were performed. "P<0.05 vs. NC. NEAT1, nuclear enriched abundant transcript 1; LV, lentivirus; sh, short hairpin RNA; miR-230a, microRNA-320a; $\mathrm{NC}$, negative control; IOD, integral optical density. 

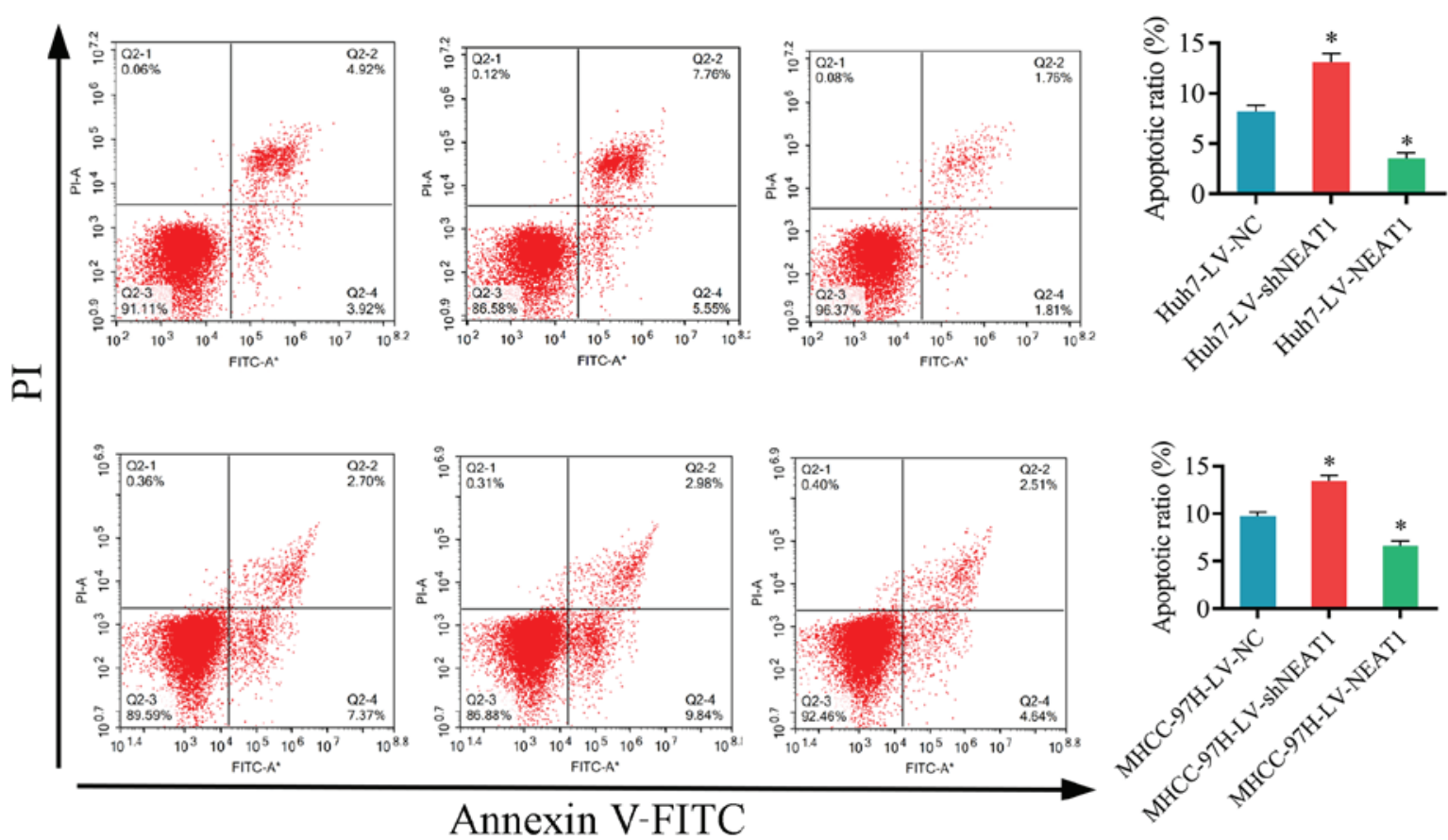

Figure 3. Effects of NEAT1 on hepatocellular carcinoma cell apoptosis. Effects of NEAT1 on the apoptosis of Huh7 and MHCC-97H cells. Cells were infected with LV-shNEAT1 or LV-NEAT1 for $48 \mathrm{~h}$. Flow cytometry was used to assess cell apoptosis. Three independent experiments were performed. ${ }^{*} \mathrm{P}<0.05 \mathrm{vs}$. NC. NEAT1, nuclear enriched abundant transcript 1; LV, lentivirus; sh, short hairpin RNA; PI, propidium iodide; NC, negative control.

Xenotransplantation of tumors. The animal experiment procedures were approved by the Animal Ethics Committee of The First Affiliated Hospital of Sun Yat-sen University (Guangzhou, China). A total of 24 1-month-old athymic female $\mathrm{BALB} / \mathrm{C}$ nude mice, with a weight range of $13-17 \mathrm{~g}$, were purchased from the Shanghai Institute of Medicine (Shanghai, China). The mice were housed at a temperature of $18-23^{\circ} \mathrm{C}$ with $40-60 \%$ humidity, a 14 -h light/10-h dark cycle, and food and water were accessible at all times. Huh7 cells $\left(2 \times 10^{5}\right)$ in $0.2 \mathrm{ml}$ normal saline transfected with LV-NEAT1, LV-shNEAT1 or LV-NC were subcutaneously implanted into the lateral abdomen of each nude mouse. A total of 24 mice were randomly divided into 4 groups; 6 mice were injected with Huh7 cells infected with LV-NEAT1, 12 were injected with Huh7 cells with LV-NC, and 6 were injected with Huh7 cells with LV-shNEAT1. In total, 2 mice died in the group of Huh7-LV-NEAT1 and Huh7-LV-NC, and finally 4 mice were analyzed. Three mice died in the group of Huh-LV-NC and Huh7-LV-shNEAT1. The volume of the tumor was estimated using a caliper once a week for 5 weeks. The nude mice were observed every $12 \mathrm{~h}$ after being inoculated with HCC cells. After 5 weeks, nude mice were anesthetized by $4 \%$ chloral hydrate intraperitoneal injection with a dose of $400 \mathrm{mg} / \mathrm{kg}$, euthanized using cervical dislocation and then the subcutaneous tumors were removed. Some mice were anesthetized and sacrificed earlier than 5 weeks if the following conditions were met: i) Maximum weight loss of nude mice was $>5 \mathrm{~g}$ in the first 2 weeks; ii) tumor volume was $<20 \mathrm{~mm}^{3}$ in the first 2 weeks; or iii) inoculated nude mice died. There were four nude mice left in the group of Huh7-LV-NEAT1 and Huh7-LV-NC and three nude mice left in the group of Huh-LV-NC and Huh7-LV-shNEAT1 at 5 weeks. The cause of death was probably due to HCC cell toxicity and poor immunity of nude mice. The weights of the tumors were measured after 5 weeks. The following formula was used to calculate tumor volume: Volume $\left(\mathrm{mm}^{3}\right)=0.5 \mathrm{x}$ length $\mathrm{x}$ width ${ }^{2}$. Multiple tumor growth was not observed in the present study. The levels of LAGE3 in the resected tumors were analyzed by immunohistochemistry.

Immunohistochemistry. Tissues were fixed with $4 \%$ formalin for $24 \mathrm{~h}$ at room temperature, embedded in paraffin and section to a thickness of $0.5-\mathrm{cm}$. Following dewaxing and rehydration, the endogenous peroxidase activity was blocked with sodium citrate buffer ( $\mathrm{pH} 6.0$ ) at $100^{\circ} \mathrm{C}$ for $20 \mathrm{~min}$, and the antigens on the slides were exposed. The slides were incubated overnight with antibodies specific for Ki67 (1:500; catalog no. ab15580; Abcam) and LAGE3 (1:100; catalog no. ab224157; Abcam) at $4^{\circ} \mathrm{C}$. Then, the slides were incubated with a rabbit anti-sheep IgG secondary antibody coupled with horseradish peroxidase (1:2000; catalog no. ab6747; Abcam) at $37^{\circ} \mathrm{C}$ for $1 \mathrm{~h}$. Following staining, nine fields of each slide were randomly selected with the help of a light microscope (magnification, x200). Ki67 and LAGE3 expression intensity was assessed by estimating the area of the fields and the IOD was calculated by the medium pixel intensity of each object. The sections were imaged with a light microscope (magnification, x200). All images were acquired and processed in TIFF format, and analysis was performed using Image ProPlus 6.0 AMS software (Media Cybernetics Inc.).

Statistical analysis. All data are presented as mean \pm standard deviation. Student's t-test was used for the analysis of two independent groups. One-way analysis of variance followed by Tukey's post hoc test was used to analyze differences among three or more groups. $\mathrm{P}<0.05$ was considered to indicate a 
A

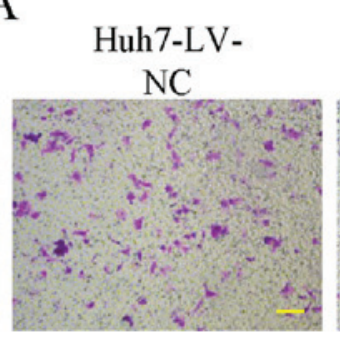

Huh7-LVshNEAT1
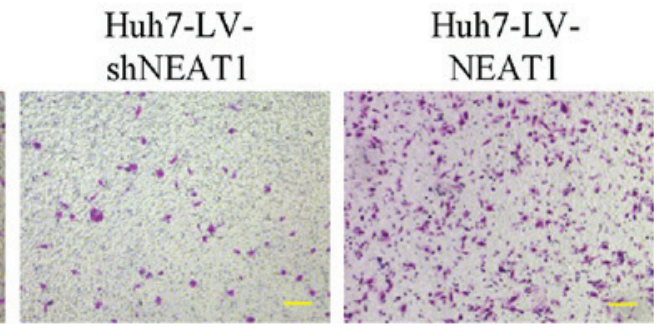

B MHCC-97H-LV$\mathrm{NC}$
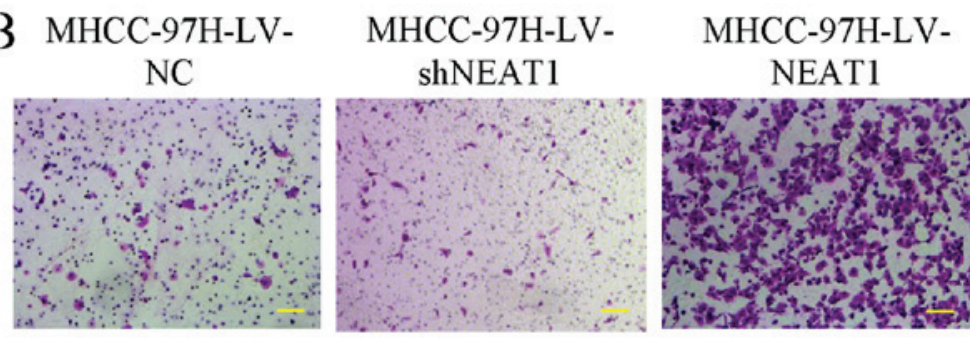

C Huh7-LV-

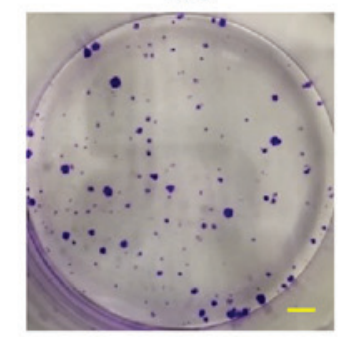

D MHCC-97H-LV-

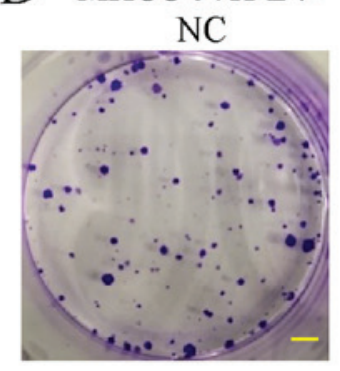

Huh7-LVshNEAT1

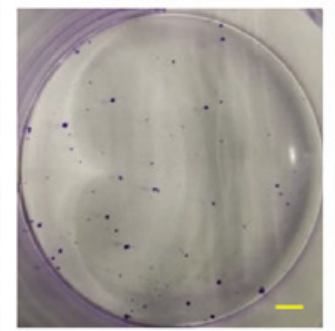

MHCC-97H-LVshNEAT1

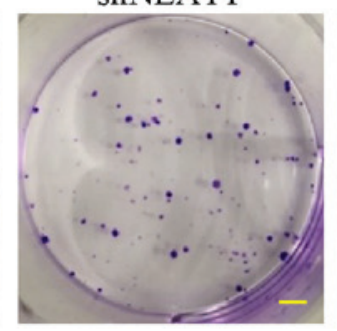

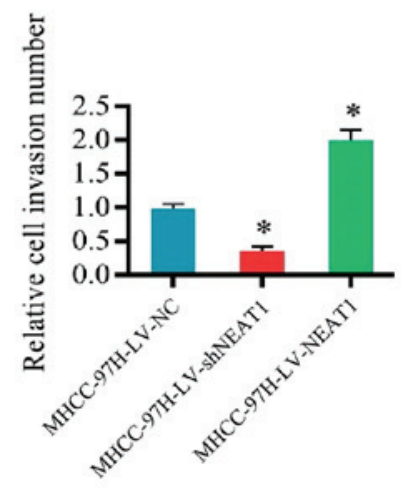
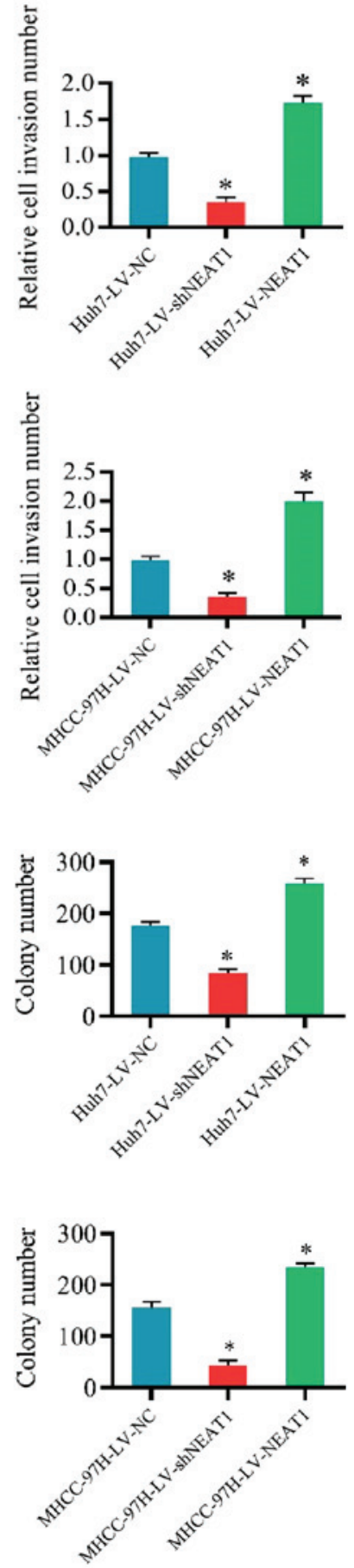

Figure 4. Effects of NEAT1 on hepatocellular carcinoma cell invasion. Cells were infected with LV-shNEAT1 or LV-NEAT1 for 48 h. Effects of NEAT1 on the invasion of (A) Huh7 and (B) MHCC-97H cells. Scale bar, $150 \mu \mathrm{m}$. A colony forming assay demonstrated the effects of the NEAT1 overexpression and silencing on colony formation in (C) Huh7 and (D) MHCC-97H cells. Scale bar, $2 \mathrm{~mm}$. Three independent experiments were performed. "P<0.05 vs. NC. NEAT1, nuclear enriched abundant transcript 1; LV, lentivirus; sh, short hairpin RNA; NC, negative control.

statistically significant difference. All statistical analysis was performed using SPSS 24.0 software (IBM Corp.).

\section{Results}

NEAT1 is upregulated and miR-320a is downregulated in HCC cells. First, NEAT1 expression was detected in the HCC cell lines Hep3B, SNU-449, SNU-398, Huh7, and MHCC-97H, the hepatoblastoma cell line Huh6 and the hepatic stellate cell line LX-2. NEAT1 expression was significantly higher in
HCC cells compared with LX-2 cells (Fig. 1A). Furthermore, miR-320a expression was significantly lower in HCC cells compared with LX-1 cells (Fig. 1B). This demonstrated that NEAT1 may be involved in the development of HCC.

Effects of NEAT1 on HCC cell proliferation. To investigate whether NEAT1 can affect HCC cell proliferation, an EdU assay was performed. Huh7 and MHCC-97H cells were infected with LV-shNEAT1 or LV-NEAT1 for $48 \mathrm{~h}$. As presented in Fig. 2A and B, NEAT1 expression was 
A Alignment NEAT1 3'-UTR-miR320a

Target: 5 ' CgGCUCGAGGGGCCAUCAGCUUUg $3^{\prime}$

$11: 1 \quad:: 11 \quad 1111111$

miRNA : 3 ' agCGGGAGAGUUGG--GUCGAAAa 5 '

B

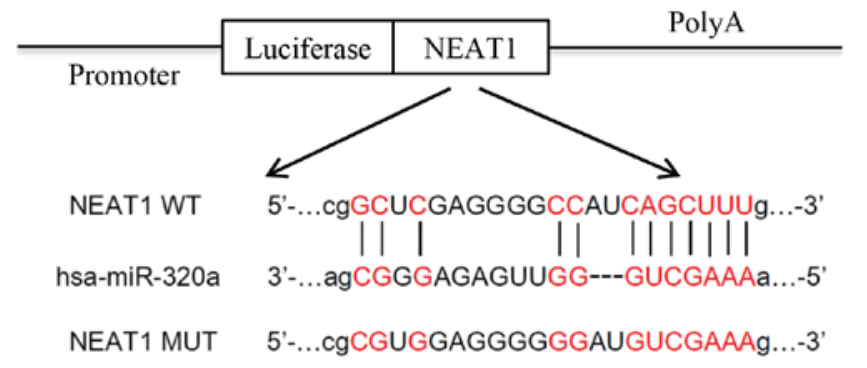

$\mathrm{C}$
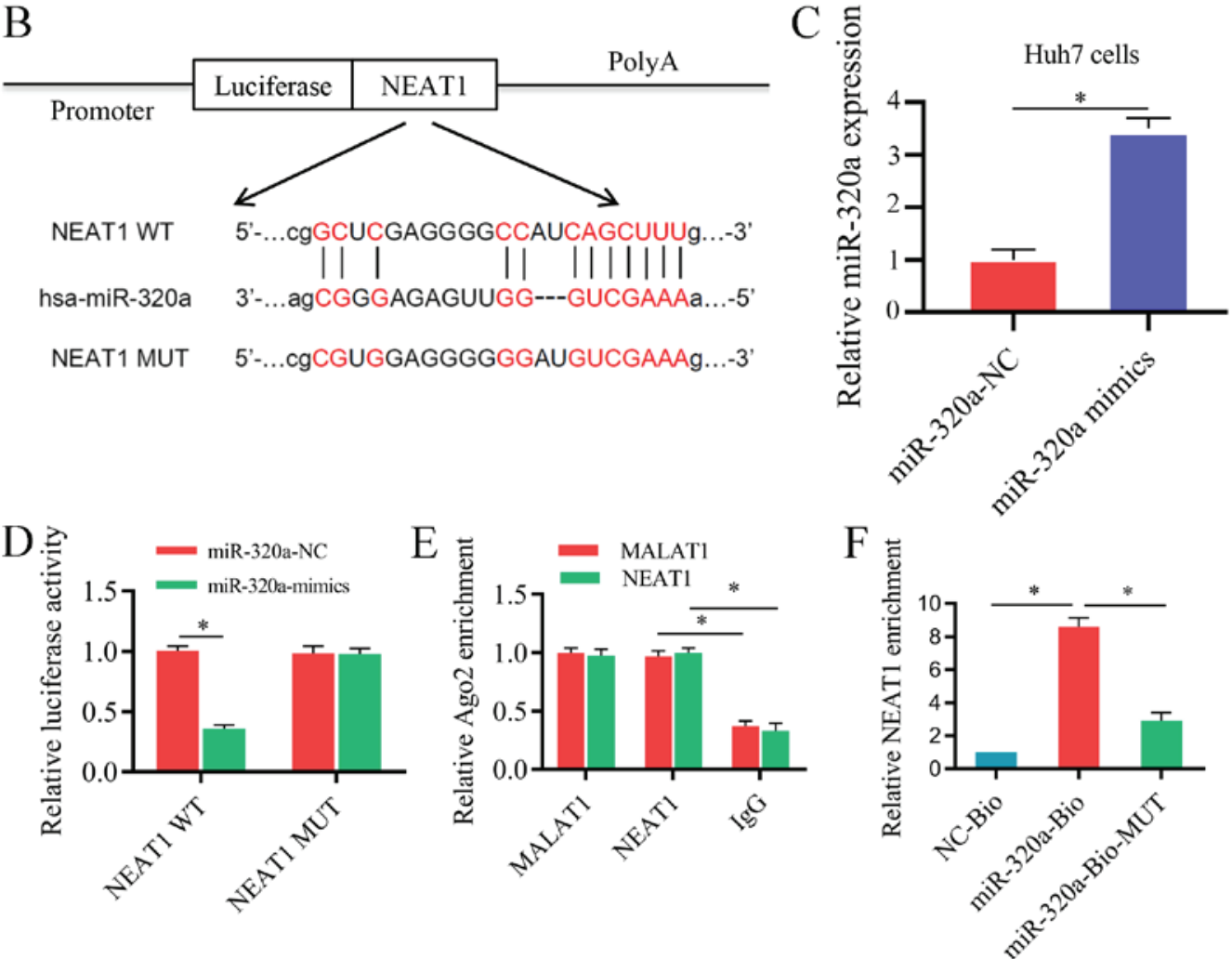

Figure 5. miR-320a serves as a target of NEAT1 in vitro. (A) The binding sites between NEAT1 and miR-320a. (B) Luciferase reporter constructs containing the NEAT1 WT or NEAT1 MUT sequence. (C) Transfection efficiency of miR-320a mimic in Huh7 cells. (D) NEAT1 WT or NEAT1 MUT were co-transfected into Huh7 cells with miR-320a mimic or the corresponding negative control. (E) The association between NEAT1 and Ago2 was tested by an RIP assay. Cellular lysates were immunoprecipitated using an Ago2 antibody or IgG. NEAT1 expression was detected by reverse transcription-quantitative PCR. The MALAT1 level was used as a positive control. (F) An RNA pull-down assay indicated the direct interaction between miR-320a and NEAT1. Cellular lysates were pulled down using NC-Bio, miR-320a-Bio or miR-320a-Bio-MUT. Three independent experiments were performed. *P<0.05. NC-Bio, biotinylated negative control; miR-320a-Bio, biotinylated miR-320a; miR-320a-Bio-MUT, miR-320a probe containing mutations in the NEAT1-binding site; WT, wild type; MUT, mutant; NEAT1, nuclear enriched abundant transcript 1; NC, negative control; 3'-UTR, 3'-untranslated region; miR-320a, microRNA-320a.

significantly reduced by LV-shNEAT1 and significantly increased by LV-NEAT1 in Huh7 and MHCC-97H cells compared with the control cells. In addition, miR-320a expression was significantly increased by LV-shNEAT1 and significantly reduced by LV-NEAT1 compared with the control (Fig. 2C and D). Next, the EdU assay was performed, which verified that Huh7 and MHCC-97H cell proliferation was significantly suppressed by reduced NEAT1 expression and significantly induced by the upregulation of NEAT1 (Fig. 2E and F). These results indicated that inhibition of NEAT1 represses the proliferation of HCC cells.

Effects of NEAT1 on HCC cell apoptosis. To investigate the effect of NEAT1 on the apoptosis of HCC cells flow cytometry was performed. The results demonstrated that the apoptosis of Huh7 and MHCC-97H cells was significantly increased by LV-shNEAT1 and significantly decreased by LV-NEAT1 compared with the control cells (Fig. 3). These results suggested that silencing NEAT1 increases HCC cell apoptosis in vitro.
Effects of NEATI on HCC cell migration. Transwell invasion assays and colony formation assays were performed to determine whether NEAT1 affects HCC cell migration and invasion. LV-shNEAT1 significantly reduced the migration capacity of Huh7 and MHCC-97H cells, while upregulation of NEAT1 significantly increased the migration (Fig. 4A and B). Subsequently, the effects of NEAT1 on colony formation were analyzed in Huh7 and MHCC-97H cells and it was identified that colony formation was significantly increased following NEAT1 upregulation (Fig. 4C and D). These results suggested that NEAT1 may promote HCC cell migration and invasion.

miR-320a acts as a target of NEAT1. LncRNAdb, StarBase and ChIPBase were used to investigate the mutual effect between NEAT1 and miR-320a. miR-320a was identified as a target of NEAT1, and the complementary binding regions were verified (Fig. 5A). To confirm these findings, a luciferase reporter assay was performed. As presented in Fig. 5B, mutations were generated in the miR-320a-binding sequence of NEAT1. miR-320a mimic was transfected into Huh7 cells and its transfection 
A Binding site of hsa-miR-320a on LAGE3:

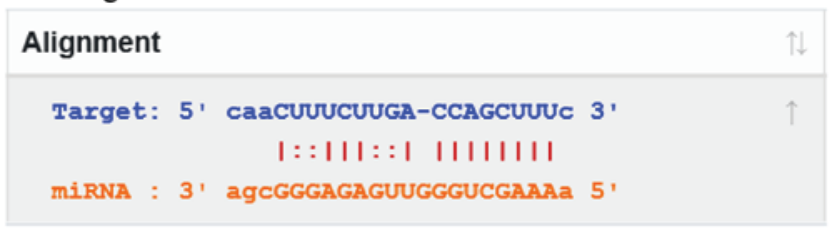

B

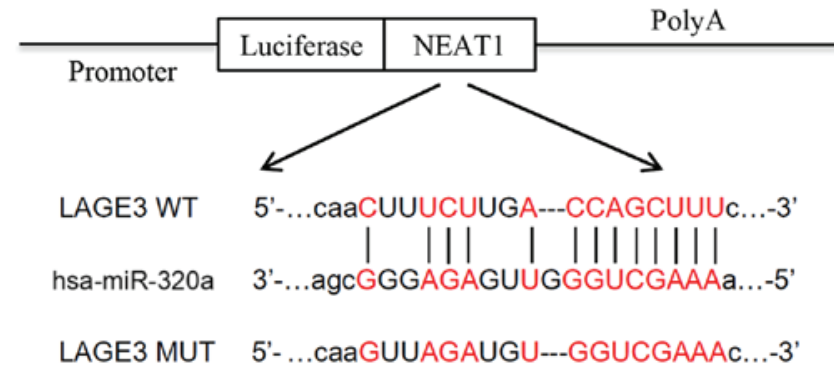

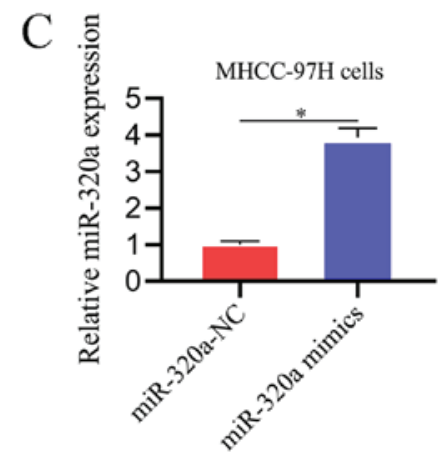
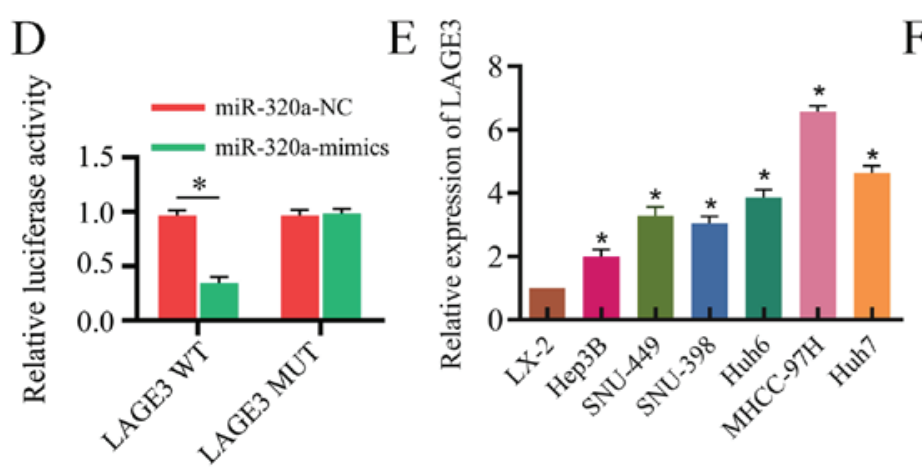

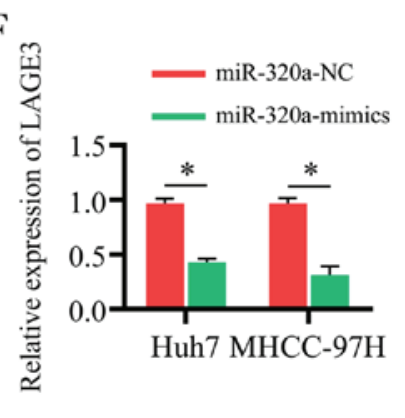

Figure 6. LAGE3 is a direct target of miR-320a. (A) Following identification of NEAT1 and miR-320a, a possible target for miR-320a in HCC carcinogenesis was identified. Starbase 3.0 aligned the complementary sites in the LAGE3 gene with miR-320a. (B) Luciferase reporter constructs containing the LAGE3 WT or mutant LAGE3 MUT sequence. (C) Transfection efficiency of miR-320a mimic in MHCC-97H cells. "P<0.05. (D) LAGE3 WT or LAGE3 MUT were co-transfected into Huh7 cells with miR-320a mimic or the corresponding NC. (E) mRNA expression of LAGE3 in HCC cells. " P $<0.05$ vs. LX-2. (F) mRNA expression of LAGE3 in Huh7 and MHCC-97H cells following transfection with miR-320a mimic or the NC for $48 \mathrm{~h}$. " $\mathrm{P}<0.05$ vs. miR-320a-NC. Three independent experiments were performed. LAGE3, L antigen family member 3; miR-320a, microRNA-320a; HCC, hepatocellular carcinoma; WT, wild type; MUT, mutant; NC, negative control; NEAT1, nuclear enriched abundant transcript 1.

efficiency is presented in in Fig. 5C. Co-transfection of a wild-type luciferase reporter plasmid with miR-320a mimic significantly reduced the reporter gene activity compared with that of the control Huh7 cells, whereas no obvious changes were observed following co-transfection with a mutant-type luciferase reporter plasmid and miR-320a mimic (Fig. 5D) due to the interaction between miR-320a mimic and NEAT-WT. Furthermore, to investigate whether NEAT1 can be coupled to miR-320a, an RIP assay was performed, and the content of NEAT1 and miR-320a in Ago2 particles was significantly higher compared with that in the IgG group in Huh7 cells (Fig. 5E). As presented in Fig. 5F, an RNA pull-down assay was performed, which revealed that significantly higher NEAT1 levels in Huh7 cells were observed with miR-320a-Bio probe compared with NC-bio or miR-320a probe. These results suggest that miR-320a is a direct target of NEAT1.

LAGE3 is a direct target of miR-320a. TargetScan, miRanda and StarBase were used for bioinformatics analysis to predict the targets of miR-320a, and LAGE3 was predicted as a target. The binding regions between miR-320a and LAGE3 are presented in Fig. 6A. The wild-type and mutant binding sites of LAGE3 are presented in Fig. 6B. The transfection efficiency of miR-320a mimic in MHCC-97H cells is presented in Fig. 6C. Wild-type luciferase reporter plasmids were co-transfected with miR-320a mimic, and the reporter activity in Huh7 cells was significantly reduced due to the interaction of miR-320 mimic and wild-type LAGE3. However, the reporter activity in Huh7 cells co-transfected with mutant-type luciferase reporter plasmids was no obviously changed (Fig. 6D). In addition, the LAGE3 mRNA expression level was revealed to be significantly higher in HCC cells and hepatoblastoma cells compared with LX-2 cells (Fig. 6E). Furthermore, the mRNA expression level of LAGE3 was significantly lower in HCC cells and hepatoblastoma cells transfected with miR-320a mimic compared with the controls (Fig. 6F).

NEAT1 regulates $H C C$ progression by modulating $\mathrm{miR}-320$ a and LAGE3 in vivo. The present study established a Huh7 cell nude mouse xenograft model to verify whether NEAT1 can regulate $\mathrm{HCC}$ in vivo. The tumor volumes and tumor weights after five weeks are presented in Fig. 7A-C. Ki-67, also known 
A
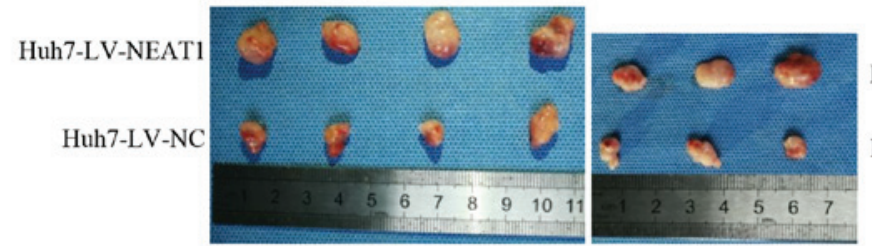

Huh7-LV-NC

Huh7-LV-shNEATI

B
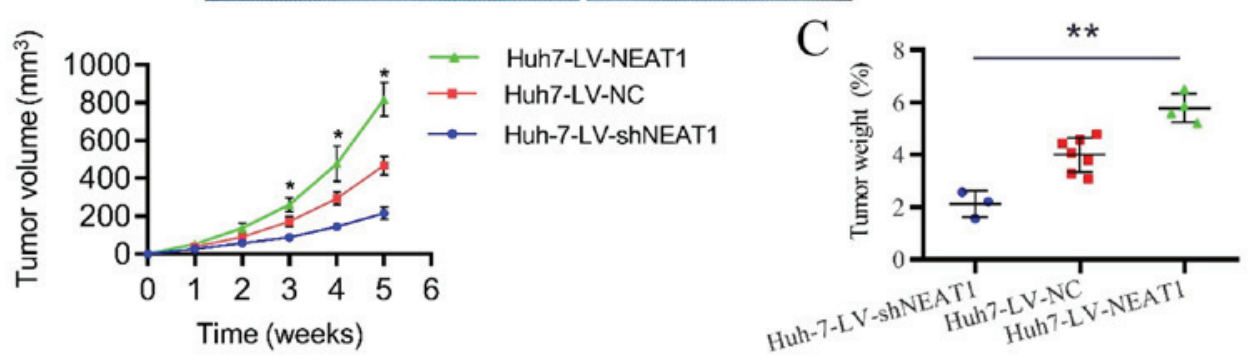

$\mathrm{D}$
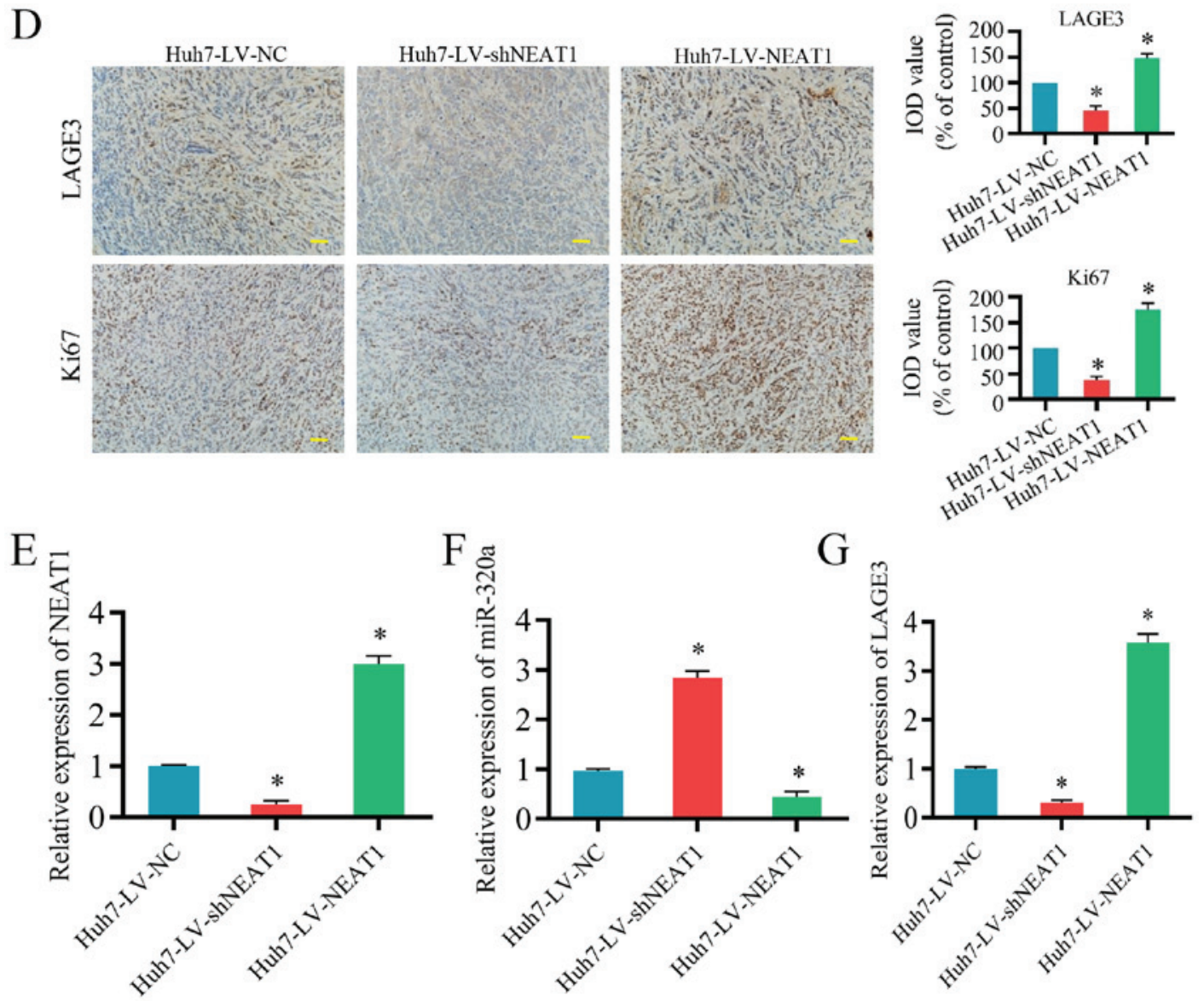

Figure 7. NEAT1 modulates hepatocellular carcinoma progression by sponging miR-320a and regulating LAGE3 in vivo. Three groups of mice were established; 12 mice were injected with Huh7 cells infected with LV-NC, six were injected with Huh7 cells infected with LV-shNEAT1, and six were injected with Huh7 cells infected with LV-NEAT1. (A) Solid tumors were collected from mouse subcutaneous tissue. Xenograft tumor (B) volume and (C) weight were shown to be significantly promoted by NEAT1. (D) Immunohistochemical staining of Ki-67 and LAGE3 in tumor tissues. Scale bar, $50 \mu \mathrm{m}$. Expression of (E) NEAT1, (F) miR-320a and (G) LAGE3 in the tumor tissues of the mice. Three independent experiments were performed. "P $<0.05$ vs. NC. NEAT1, nuclear enriched abundant transcript 1; miR-320a, microRNA-320a; LV, lentivirus; NC, negative control; sh, short hairpin RNA; LAGE3, L antigen family member 3; IOD, integral optical density.

as antigen $\mathrm{Ki}-67$ or MKI67, is a protein encoded by the MKI67 gene in mammals. The expression of Ki67 is strongly associated with tumor cell proliferation and growth and is widely used as a proliferation marker in routine pathological investigations $(27,28)$. As presented in Fig. 7D, immunohistochemistry revealed that LV-shNEAT1 significantly inhibited LAGE3 and Ki-67, while LV-NEAT1 significantly increased expression of LAGE3 and Ki-67. RT-qPCR was performed to analyze the expression level of NEAT1, miR-320a and LAGE3. As presented in Fig. 7E-G, when the expression of NEAT1 was downregulated, the expression of miR-320a was significant increased, while the expression level of LAGE3 was significantly reduced. By contrast, when NEAT1 was overexpressed, the effects of miR-320a and LAGE3 expression were reversed. It was identified that NEAT1 overexpression promoted LAGE3 expression via sponging miR-320a in vivo, 
while the downregulation of NEAT1 demonstrated the opposite effect. These results revealed that NEAT1 promotes the development of HCC by targeting miR-320a and LAGE3 in vivo.

\section{Discussion}

In the present study, NEAT1 expression was demonstrated to be significantly higher in HCC cells. NEAT1-silencing could restrain the progression of HCC, while NEAT1 overexpression was capable of promoting HCC development by regulating miR-320a both in vitro and in vivo. Further mechanistic studies indicated that LAGE3 was a direct target of miR-320a. In conclusion, it was demonstrated that NEAT1 acts as a competitive endogenous RNA (ceRNA) for miR-320a to enhance LAGE3 expression in the tumorigenesis of HCC.

It is widely acknowledged that only $\sim 1 \%$ of the genome encodes proteins, while the vast majority of the transcribed human genome consists of noncoding sequences (29). The lncRNA NEAT1 is among these sequences. NEAT1 is located in the nucleus, is a highly abundant lncRNA with a length of $\sim 4 \mathrm{~kb}$ and acts as a critical component for the structure of paraspeckles $(30,31)$. The present study observed that the expression of NEAT1 was enhanced in HCC cell lines compared with the hepatic stellate cells LX-2. These results suggest that NEAT1 may serve a role in tumor carcinogenesis in HCC. To investigate the role of NEAT1 in HCC, its expression was downregulated and upregulated through stable transfection of Huh7 and MHCC-97H cells. Silencing NEAT1 significantly inhibited proliferation and migration but increased apoptosis of HCC cells. By contrast, the overexpression of NEAT1 significantly promoted proliferation and migration but inhibited the apoptosis of HCC cells. In addition, the downregulation of NEAT1 reduced tumor weight and volume in xenograft nude mice models, while overexpression of NEAT1 resulted in the opposite effects. These results indicate that NEAT1 is an oncogene in HCC.

The present study further investigated the potential mechanism of NEAT1 in the development of HCC. miR-320a is a crucial tumor suppressor in various neoplasms, including lung cancer, salivary adenoid cystic carcinoma, breast cancer and endometrial carcinoma (32-35). Notably, Zhang et al (36) reported that miR-320a is downregulated in HCC cell lines and tissues. Overexpression of miR-320a not only inhibited the proliferation and invasion of HCC cells but also decreased tumor growth in vivo. In line with this study, the present study also observed a significant decrease in the expression of miR-320a in HCC cell lines. Bioinformatics analysis suggested that NEAT1 contains a binding region for miR-320a. Therefore, the association between NEAT1 and miR-320a in HCC was investigated. In the present study, double luciferase reporter analysis and RNA pull-down assays confirmed that NEAT1 could bind directly to miR-320a in HCC cells. Silencing NEAT1 elevated miR-320a expression in both HCC cells and xenograft nude mice models. By contrast, overexpression of NEAT1 decreased miR-320a expression. These results indicated that NEAT1 acts by negatively modulating miR-320a expression in the development of HCC.

LAGE3 (also known as ESO3), a subunit of the EKC/KEOPS complex, is evolutionarily conserved and ubiquitously expressed in somatic tissues $(37,38)$. It has been reported that the EKC/KEOPS complex, the matrix of LAGE3, is associated with the processes of transcription, protein synthesis, telomere homeostasis, genomic instability and cell growth $(39,40)$. LAGE3 interacts with a frequently overexpressed human tumor antigen, preferably expresses antigen in melanoma, and may engage in neoplastic processes (41). However, the role of LAGE3 is still not fully understood. The present study found that LAGE3 is a target of miR-320a and that miR-320a negatively regulates LAGE3 expression in HCC cells. LAGE3 was elevated in HCC cell lines compared with normal liver cell lines. LAGE3 may participate in the oncogenesis of HCC and serve as a potential therapeutic target.

It has been reported that certain IncRNAs can act as sponges for miRNAs by modulating the expression of miRNA target genes in various human diseases, including cancer. For example, the lncRNA TUG1 has been reported to promote the development of oral squamous cell carcinoma via sponging miR-524-5p, thereby affecting distal-less homeobox 1 expression by acting as a ceRNA (42). Furthermore, IncRNA LINC01234 promoted the proliferation and the occurrence of gastric carcinoma by sponging miR-204-5p as a ceRNA and alleviating the suppression of the target gene core-binding factor subunit $\beta$ (14). Through bioinformatics analysis, the present study identified that NEAT1 contains similar response elements as LAGE3 at the putative miR-320a binding sites. This suggests that NEAT1 and LAGE3 may compete for the same binding site of miR-320a. Therefore, it was hypothesized that NEAT1 may play a carcinogenic role by sponging miR-320a and reducing the suppression of LAGE3 in HCC. Then, an anti-AGO2 RIP assay was conducted, and the results revealed that the content of NEAT1 and mir-320a in Ago2 particles was higher than compared with that in the IgG group. Silencing NEAT1 significantly decreased the expression of LAGE3 in mouse HCC tumor tissues, and overexpression of NEAT1 had the opposite effect. Collectively, these results indicated that NEAT1 functions as a ceRNA for miR-320a to modulate LAGE3 expression.

In conclusion, the present study demonstrated that NEAT1 may facilitate the progression of HCC. In the current study, the potential mechanism of the NEAT1/miR-320a/LAGE3 axis in HCC cells was confirmed, and the overexpression of NEAT1 was found to promote the progression of HCC. In addition, negative correlation association between NEAT1 and miRNA-320a was identified. The present study focused on LAGE3 since it is targeted by miR-320a. The current findings reveal that the NEAT1/miR-320a/LAGE3 axis participates in the development of HCC and that NEAT1 may be a potential biomarker for HCC.

\section{Acknowledgments}

Not applicable.

\section{Funding}

This study was supported by the National Natural Science Foundation of China (grant nos. 81401324 and 81770410), Guangdong Provincial International Cooperation Base of Science and Technology (Organ Transplantation) (grant 
no. 2015B050501002), Guangdong Provincial Natural Science Funds for Distinguished Young Scholars (grant no. 2015A030306025), Special Support Program for Training High-Level Talent in Guangdong Province (grant no. 2015TQ01R168), Pearl River Nova Program of Guangzhou (grant no. 201506010014), and Scientific Program for Young Teachers of Sun Yat-sen University (grant no. 16ykpy05).

\section{Availability of data and materials}

The data used and analyzed in this study are available from the corresponding author upon request.

\section{Authors' contributions}

YZ and MC contributed to the design and writing of the study. ZZhu and $\mathrm{CH}$ acquired and analyzed the data. $\mathrm{CH}, \mathrm{YT}, \mathrm{SH}$, LW, HC and CS performed the experiments. MC, ZZha and WJ contributed to the data analysis. YZ, YH and XQ read and revised the manuscript, and made substantial contributions to the interpretation and analysis of the data. $\mathrm{YZ}$ and $\mathrm{XQ}$ contributed to drafting the manuscript, critically modifying important content, and approving the version to be published. All authors read and approved the final manuscript.

\section{Ethics approval and consent to participate}

All experimental procedures and protocols were approved by the Research Ethics Committee on the Ethics of Animal Experiments of The First Affiliated Hospital of Sun Yat-Sen University (Guangzhou, China).

\section{Patient consent for publication}

Not applicable.

\section{Competing interests}

The authors declare that they have no competing interests.

\section{References}

1. Bray F, Ferlay J, Soerjomataram I, Siegel RL, Torre LA and Jemal A: Global cancer statistics 2018: GLOBOCAN estimates of incidence and mortality worldwide for 36 cancers in 185 countries. CA Cancer J Clin 68: 394-424, 2018.

2. Sia D, Villanueva A, Friedman SL and Llovet JM: Liver cancer cell of origin, molecular class, and effects on patient prognosis Gastroenterology 152: 745-761, 2017.

3. Villanueva A: Hepatocellular carcinoma. N Engl J Med 380: 1450-1462, 2019.

4. $\mathrm{Xu}$ J: Trends in liver cancer mortality among adults aged 25 and over in the United States, 2000-2016. NCHS Data Brief: 1-8, 2018.

5. Necsulea A, Soumillon M, Warnefors M, Liechti A, Daish T, Zeller U, Baker JC, Grützner F and Kaessmann H: The evolution of lncRNA repertoires and expression patterns in tetrapods. Nature 505: 635-640, 2014

6. Fernandes JCR, Acuña SM, Aoki JI, Floeter-Winter LM and Muxel SM: Long non-coding RNAs in the regulation of gene expression: Physiology and disease. Noncoding RNA 5: 17, 2019.

7. Bartel DP: Metazoan MicroRNAs. Cell 173: 20-51, 2018.

8. Friedman RC, Farh KKH, Burge CB and Bartel DP: Most mammalian mRNAs are conserved targets of microRNAs. Genome Res 19: 92-105, 2009.
9. Kabekkodu SP, Shukla V, Varghese VK, D' Souza J, Chakrabarty S and Satyamoorthy K: Clustered miRNAs and their role in biological functions and diseases. Biol Rev 93: 1955-1986, 2018.

10. Marchese FP, Raimondi I and Huarte M: The multidimensional mechanisms of long noncoding RNA function. Genome Biol 18: 206, 2017

11. Ding L, Ren J, Zhang D, Li Y, Huang X, Hu Q, Wang H, Song Y, Ni Y and Hou Y: A novel stromal lncRNA signature reprograms fibroblasts to promote the growth of oral squamous cell carcinoma via LncRNA-CAF/interleukin-33. Carcinogenesis 39: 397-406, 2018.

12. Xing F, Liu Y, Wu SY, Wu K, Sharma S, Mo YY, Feng J, Sanders S, Jin G, Singh R, et al: Loss of XIST in breast cancer activates MSN-c-Met and reprograms microglia via exosomal miRNA to promote brain metastasis. Cancer Res 78: 4316-4330, 2018.

13. Yang Y, Zhang JP, Chen X, Xu X, Cao G, Li H and Wu T: LncRNA FTX sponges miR-215 and inhibits phosphorylation of vimentin for promoting colorectal cancer progression. Gene Ther 25: 321-330, 2018.

14. Chen X, Chen Z, Yu S, Nie F, Yan S, Ma P, Chen Q, Wei C, $\mathrm{Fu} \mathrm{H}, \mathrm{Xu}$ T, et al: Long noncoding RNA LINC01234 functions as a competing endogenous RNA to regulate CBFB expression by sponging miR-204-5p in gastric cancer. Clin Cancer Res 24: 2002-2014, 2018

15. Zhao L, Zhang YJ and Zhang YB: Long noncoding RNA CASC2 regulates hepatocellular carcinoma cell oncogenesis through miR-362-5p/Nf-B axis. J Cell Physiol 233: 6661-6670, 2018.

16. Yang X, Qu S, Wang L, Zhang H, Yang Z, Wang J, Dai B, Tao K, Shang R, Liu Z, et al: PTBP3 splicing factor promotes hepatocellular carcinoma by destroying the splicing balance of NEAT1 and pre-miR-612. Oncogene 37: 6399-6413, 2018.

17. Liu Z, Chang Q, Yang F, Liu B, Yao HW, Bai ZG, Pu CS, Ma XM, Yang Y, Wang TT, et al: Long non-coding RNA NEAT 1 overexpression is associated with unfavorable prognosis in patients with hepatocellular carcinoma after hepatectomy: A Chinese population-based study. Eur J Surg Onc 43: 1697-1703, 2017.

18. Chen S and Xia X: Long noncoding RNA NEAT1 suppresses sorafenib sensitivity of hepatocellular carcinoma cells via regulating miR-335-c-Met. J Cell Physiol: Apr 1, 2019 (Epub ahead of print).

19. Müller V, Oliveira-Ferrer L, Steinbach B, Pantel K and Schwarzenbach H: Interplay of lncRNA H19/miR-675 and lncRNA NEAT1/miR-204 in breast cancer. Mol Oncol 13: 1137-1149, 2019.

20. Kong X, Zhao Y, Li X, Tao Z, Hou M and Ma H: Overexpression of HIF-2 $\alpha$-dependent NEAT1 promotes the progression of non-small cell lung cancer through miR-101-3p/SOX $9 / \mathrm{Wnt} / \beta$-catenin signal pathway. Cell Physiol Biochem 52: 368-381, 2019.

21. Zhang M, Weng WW, Zhang QY, Wu Y, Ni S, Tan C, Xu M, Sun H, Liu C, Wei P and Du X: The lncRNA NEAT1 activates $\mathrm{Wnt} / \beta$-catenin signaling and promotes colorectal cancer progression via interacting with DDX5. J Hematol Oncol 11: 113, 2018.

22. Yan P, Su Z, Zhang Z and Gao T: LncRNA NEAT1 enhances the resistance of anaplastic thyroid carcinoma cells to cisplatin by sponging miR-9-5p and regulating SPAG9 expression. Int J Oncol 55: 988-1002, 2019.

23. Xia TF, Chen J, Wu K, Zhang J and Yan Q: Long noncoding RNA NEAT1 promotes the growth of gastric cancer cells by regulating miR-497-5p/PIK3R1 axis. Eur Rev Med Pharmacol Sci 23: 6914-6926, 2019.

24. He J, Xu F, Man X, Zhang Y and Li H: Long non-coding RNA NEAT1 promotes tumor development and metastasis through targeting RAB9A in malignant melanoma. Minerva Med: Jul 17 , 2019 doi: 10.23736/S0026-4806.19.06235-9. Online ahead of print.

25. Livak KJ and Schmittgen TD: Analysis of relative gene expression data using real-time quantitative PCR and the 2(-Delta Delta C(T)) method. Methods 25: 402-408, 2001

26. Sun JY, Zhao ZW, Li WM, Yang G, Jing PY, Li P, Dang HZ, Chen Z, Zhou YA and Li XF: Knockdown of MALAT1 expression inhibits HUVEC proliferation by upregulation of miR-320a and downregulation of FOXM1 expression. Oncotarget 8: 61499-61509, 2017.

27. Scholzen T and Gerdes J: The Ki-67 protein: From the known and the unknown. J Cell Physiol 182: 311-322, 2000. 
28. Brown DC and Gatter KC: Ki67 protein: The immaculate deception? Histopathology 40: 2-11, 2002.

29. Ponting CP, Oliver PL and Reik W: Evolution and functions of long noncoding RNAs. Cell 136: 629-641, 2009.

30. Clemson CM, Hutchinson JN, Sara SA, Ensminger AW, Fox AH, Chess A and Lawrence JB: An architectural role for a nuclear noncoding RNA: NEAT1 RNA is essential for the structure of paraspeckles. Mol Cell 33: 717-726, 2009.

31. Souquere S, Beauclair G, Harper F, Fox A and Pierron G: Highly ordered spatial organization of the structural long noncoding NEAT1 RNAs within paraspeckle nuclear bodies. Mol Biol Cell 21: 4020-4027, 2010.

32. Fortunato O, Borzi C, Milione M, Centonze G, Conte D, Boeri M, Verri C, Moro M, Facchinetti F, Andriani F, et al: Circulating mir-320a promotes immunosuppressive macrophages M2 phenotype associated with lung cancer risk. Int J Cancer 144: 2746-2761, 2019.

33. Sun L, Liu B, Lin Z, Yao Y, Chen Y, Li Y, Chen J, Yu D, Tang Z, Wang B, et al: miR-320a acts as a prognostic factor and Inhibits metastasis of salivary adenoid cystic carcinoma by targeting ITGB3. Mol Cancer 14: 96, 2015.

34. Yu J, Wang JG, Zhang L, Yang HP, Wang L, Ding D, Chen Q, Yang WL, Ren KH, Zhou DM, et al: MicroRNA-320a inhibits breast cancer metastasis by targeting metadherin. Oncotarget 7 38612-38625, 2016.

35. Shu S, Liu X, Xu M, Gao X, Chen S, Zhang L and Li R: MicroRNA-320a acts as a tumor suppressor in endometrial carcinoma by targeting IGF-1R. Int J Mol Med 43: 1505-1512, 2019.

36. Zhang Z, Li X, Sun W, Yue S, Yang J, Li J, Ma B, Wang J, Yang X, $\mathrm{Pu}$ M, et al: Loss of exosomal miR-320a from cancer-associated fibroblasts contributes to $\mathrm{HCC}$ proliferation and metastasis. Cancer Lett 397: 33-42, 2017.
37. Alpen B, Güre AO, Scanlan MJ, Old LJ and Chen YT: A new member of the NY-ESO-1 gene family is ubiquitously expressed in somatic tissues and evolutionarily conserved. Gene 297: 141-149, 2002.

38. Wan LC, Maisonneuve P, Szilard RK, Lambert JP, Ng TF, Manczyk N, Huang H, Laister R, Caudy AA, Gingras AC, et al: Proteomic analysis of the human KEOPS complex identifies C14ORF142 as a core subunit homologous to yeast Gon7. Nucleic Acids Res 45: 805-817, 2017.

39. Daugeron MC, Lenstra TL, Frizzarin M, El Yacoubi B, Liu X, Baudin-Baillieu A, Lijnzaad P, Decourty L, Saveanu C, Jacquier A, et al: Gcn4 misregulation reveals a direct role for the evolutionary conserved EKC/KEOPS in the t6A modification of tRNAs. Nucleic Acids Res 39: 6148-6160, 2011.

40. Rojas-Benitez D, Ibar C and Glavic A: The Drosophila EKC/KEOPS complex: Roles in protein synthesis homeostasis and animal growth. Fly (Austin) 7: 168-172, 2013.

41. Costessi A, Mahrour N, Sharma V, Stunnenberg R, Stoel MA, Tijchon E, Conaway JW, Conaway RC and Stunnenberg HG: The human EKC/KEOPS complex is recruited to Cullin2 ubiquitin ligases by the human tumour antigen PRAME. PLoS One 7: e42822, 2012.

42. Liu S, Liu LH, Hu WW and Wang M: Long noncoding RNA TUG1 regulates the development of oral squamous cell carcinoma through sponging miR-524-5p to mediate DLX1 expression as a competitive endogenous RNA. J Cell Physiol 234: 20206-20216, 2019. 